stärkere Schmerzreduktion als mit $300 \mathrm{mg}$ Pregabalin täglich erreicht $(p<0,001)$. Nach acht Wochen war bei $52 \%$ der Patienten der Duloxetin-Gruppe eine Schmerzreduktion von mehr als $30 \%$ eingetreten (vs. bei 36,9\% in der Pregabalin-Gruppe, $p<0,001$ ). Bei $40,3 \%$ der Patienten der Duloxetin-Gruppe nahmen die Schmerzen sogar um mehr als $50 \%$ ab (vs. bei $27,8 \%$ in der PregabalinGruppe, $\mathrm{p}<0,001)$.

Bei der intensivierten Therapie der NonResponder ergab die gepoolte Analyse nach weiteren acht Wochen keine Unterschiede in der Schmerzreduktion zwischen der Kombinationstherapie und der Hochdosis-Monotherapie, betonte Kern $(p=0,37)$. Auch bei der Verbesserung der funktionellen schmerzbedingten Beeinträchtigungen, etwa der allgemeinen Aktivität, des
Gehvermögens oder des Schlafes, schnitt die Initialtherapie mit Duloxetin signifikant besser ab als die Initialtherapie mit Pregabalin.

Dagmar Jäger-Becker

1. www.versorgungsleitlinien.de/diabetes $2 /$ dm2_neuro/pdf/nvl-t2d-neuro-lang.pdf

2. Diener HC, Putzki N (Hrsg). Thieme Verlag, 4. Auflage 2008; 630-39

3. www.nice.org.uk/guidance/CG96

4. Wilhelm $S$ et al., 14. Weltschmerzkongress IASP, Mailand 2012; Poster 137

Pressegespräch „Sind zwei wirklich besser als eins? Neue Daten aus der bisher größten rando misierten, doppelblinden Kombinationsstudie mit Duloxetin und Pregabalin", DGSS,

Mannheim, 18.10.2012

Veranstalter: Lilly

\title{
Epilepsie - was sich durch eine Kombinationstherapie erreichen lässt
}

— Durch eine Add-on-Therapie mit einem Antiepileptikum der zweiten Generation haben Epilepsiepatienten, die auf eine Monotherapie nicht oder nicht ausreichend angesprochen haben, eine hohe Chance anfallsfrei zu werden.

Ein Großteil aller Epilepsiepatienten wird durch eine Monotherapie nicht anfallsfrei. Eine Kombinationsbehandlung könne insbesondere bei schwierigen Epilepsieformen dazu beitragen, die Anfallskontrolle zu verbessern, berichtete Professor Andreas Schulze-Bonhage vom Universitätsklinikum Freiburg. Durch die Ausnutzung günstiger pharmakokinetischer Eigenschaften und supraadditiver Wirkungen verschiedener Antikonvulsiva könne eine Add-on-Therapie mit modernen Wirkstoffen wie Lacosamid (Vimpat ${ }^{\oplus}$ ) die Anfallskontrolle erheblich verbessern. Die Substanz weise ein günstiges Verträglichkeitsprofil auf und zeige keine relevanten Interaktionen mit anderen Medikamenten, einschließlich anderer Antiepileptika und oraler Kontrazeptiva.

Die gepoolten Daten der drei Zulassungsstudien bei insgesamt 1.308 Patienten mit fokalen Anfällen zeigen, dass sowohl Wirksamkeit als auch Verträglichkeit von Lacosamid deutlich verbessert werden können, wenn die Substanz mit Nicht-Natriumkanal-
Blockern kombiniert wird [Sake J-K et al. CNS Drugs 2010; 24: 1055-68]. Aber auch zu klassischen Natriumkanal-Blockern kann Lacosamid hinzugegeben werden. Dann sei es aber sinnvoll, die Dosis der Komedikation zu reduzieren, um die „drug-load“ zu verringern, riet Dr. Stefan Stodieck vom Evangelischen Krankenhaus Alsterdorf in Hamburg.

Die Interimsdaten der prospektiven Beobachtungsstudie VITOBA („Vimpat Added to One Baseline $A E D^{\prime \prime}$ ) bestätigen, dass die Add-on-Therapie mit Lacosamid auch unter den Bedingungen des Praxisalltags effektiv und gut verträglich ist [Noack-Rink $M$ et al. Poster UCB Scientific Exhibit, $65^{\text {th }}$ Annual Meeting of the American Epilepsy Society, Baltimore, 2011]. Nach sechs Monaten wurde die Anfallsfrequenz bei drei Viertel der Patienten um $\geq 50 \%$ reduziert; $43,4 \%$ wurden anfallsfrei. Wenn die Substanz in Kombination zur ersten Monotherapie verabreicht wurde, erreichten sogar $67 \%$ der Patienten Anfallsfreiheit.

Abdol A. Ameri

Presse-Round-Table "Zukunft der Epilepsietherapie: Welchen Beitrag leistet Lacosamid zum Fortschritt?" München, 26.1.2013, München Veranstalter UCB Pharma

\section{Interferon beta -1a bald pegyliert?}

Zur zwei- oder vierwöchentlichen Verabreichung von Peginterferon beta-1a für die Behandlung von Patienten mit schubförmiger multipler Sklerose (RRMS) liegen eine erste Wirksamkeitsanalyse sowie Sicherheitsdaten aus der zulassungsrelevanten klinischen PhaseIII-Studie ADVANCE vor.

Peginterferon beta-1a ist ein pegyliertes Interferon beta-1a, dessen Halbwertszeit und damit die Dauer der Wirkstoffexposition im Körper verlängert wurde. Unter beiden Behandlungsregimen von Peginterferon beta-1a zeigte sich in ADVANCE eine signifikante Reduktion der jährlichen Schubrate nach einem Jahr, um $35,6 \%$ beziehungsweise $27,5 \%$. Auch in der Reduktion der Behinderungsprogression und dem Anteil der Patienten mit Schüben sowie den MRT-Parametern waren beide Dosierungen gegenüber Placebo überlegen und wiesen ein günstiges Sicherheits- und Verträglichkeitsprofil auf.

Bei Zulassung könnte Peginterferon beta-1a für MS-Patienten eine Therapie mit einer noch geringeren Applikationsfrequenz ermöglichen, als bisher unter INF beta-1-a, die gleichzeitig eine Reduktion von Schüben und Behinderungsprogression in Aussicht stellt.

Nach Informationen von Biogen Idec

\section{Wearing-off beim idiopathischen Parkinsonsyndrom}

Ein Wearing-off ist in relativ frühen Stadien des idiopathischen Parkinsonsyndroms nicht nur eine häufige, sondern oft auch stark belastende Komplikation der Therapie.

Zur Behandlung des Wearing-off steht das mit dem COMT-Hemmer Entacapon pharmakokinetisch optimierte Levodopa/Carbidopa (LCE) zur Verfügung. Mit LCE in einer Tablette (Stalevo ${ }^{\circledR}$ ) wird dem Patienten die tägliche Einnahme und dadurch die Therapieadhärenz erleichtert. LCE steht in sieben Dosisstärken zur Verfügung, so dass die Therapie flexibel und präzise auf die Bedürfnisse des einzelnen Patienten angepasst werden kann. Es kann nicht zuletzt dazu beitragen, höhere LevodopaTagesdosen, die das Risiko motorischer Komplikationen steigern, zu vermeiden.

Nach Informationen von Orion Pharma 